\title{
Video Article \\ Quantitative Visualization and Detection of Skin Cancer Using Dynamic Thermal Imaging
}

\author{
Cila Herman ${ }^{1}$, Muge Pirtini Cetingul ${ }^{1}$ \\ ${ }^{1}$ Department of Mechanical Engineering, The Johns Hopkins University \\ Correspondence to: Cila Herman at cherman@jhu.edu \\ URL: https://www.jove.com/video/2679 \\ DOI: doi:10.3791/2679
}

Keywords: Medicine, Issue 51, Infrared imaging, quantitative thermal analysis, image processing, skin cancer, melanoma, transient thermal response, skin thermal models, skin phantom experiment, patient study

Date Published: 5/5/2011

Citation: Herman, C., Pirtini Cetingul, M. Quantitative Visualization and Detection of Skin Cancer Using Dynamic Thermal Imaging. J. Vis. Exp. (51), e2679, doi:10.3791/2679 (2011).

\section{Abstract}

In 2010 approximately 68,720 melanomas will be diagnosed in the US alone, with around 8,650 resulting in death ${ }^{1}$. To date, the only effective treatment for melanoma remains surgical excision, therefore, the key to extended survival is early detection ${ }^{2,3}$. Considering the large numbers of patients diagnosed every year and the limitations in accessing specialized care quickly, the development of objective in vivo diagnostic instruments to aid the diagnosis is essential. New techniques to detect skin cancer, especially non-invasive diagnostic tools, are being explored in numerous laboratories. Along with the surgical methods, techniques such as digital photography, dermoscopy, multispectral imaging systems (MelaFind), laser-based systems (confocal scanning laser microscopy, laser doppler perfusion imaging, optical coherence tomography), ultrasound, magnetic resonance imaging, are being tested. Each technique offers unique advantages and disadvantages, many of which pose a compromise between effectiveness and accuracy versus ease of use and cost considerations. Details about these techniques and comparisons are available in the literature ${ }^{4}$.

Infrared (IR) imaging was shown to be a useful method to diagnose the signs of certain diseases by measuring the local skin temperature. There is a large body of evidence showing that disease or deviation from normal functioning are accompanied by changes of the temperature of the body, which again affect the temperature of the skin ${ }^{5,6}$. Accurate data about the temperature of the human body and skin can provide a wealth of information on the processes responsible for heat generation and thermoregulation, in particular the deviation from normal conditions, often caused by disease. However, IR imaging has not been widely recognized in medicine due to the premature use of the technology ${ }^{7,8}$ several decades ago, when temperature measurement accuracy and the spatial resolution were inadequate and sophisticated image processing tools were unavailable. This situation changed dramatically in the late 1990s-2000s. Advances in IR instrumentation, implementation of digital image processing algorithms and dynamic IR imaging, which enables scientists to analyze not only the spatial, but also the temporal thermal behavior of the skin $^{9}$, allowed breakthroughs in the field.

In our research, we explore the feasibility of IR imaging, combined with theoretical and experimental studies, as a cost effective, non-invasive, in vivo optical measurement technique for tumor detection, with emphasis on the screening and early detection of melanoma ${ }^{10-13}$. In this study, we show data obtained in a patient study in which patients that possess a pigmented lesion with a clinical indication for biopsy are selected for imaging. We compared the difference in thermal responses between healthy and malignant tissue and compared our data with biopsy results. We concluded that the increased metabolic activity of the melanoma lesion can be detected by dynamic infrared imaging.

\section{Video Link}

The video component of this article can be found at https://www.jove.com/video/2679/

\section{Protocol}

\section{Setup}

1. A temperature controlled exam room equipped with an infrared camera and a PC for infrared image acquisition and storage as well as a data acquisition card connected to a computer are shown in Fig.1a.

2. The room temperature and skin surface temperature are monitored by thermocouples attached to a data acquisition card during the patient study and measurement data are stored on the computer.

\section{Image Acquisition}

1. Since the lesion cannot be detected in the thermal image without the cooling effect, a square adhesive marker is used to localize the pigmented lesion of interest and its surroundings (Fig. 1b).

2. We acquire a bright light image of the pigmented lesion and the adhesive window with a digital camera (Canon PowerShot G11) (Fig. 1b). 
3. A dermatoscope connected to a digital camera (DermLite Foto System) is used to capture the polarized light image.

4. We acquire a steady state infrared image with a Merlin midwave $(3-5 \mu \mathrm{m})$ infrared camera shown in Fig.1a, c.

5. We apply a stream of cold air to the area of the patient's skin containing the lesion as well as a $50 \mathrm{~mm}$ diameter surrounding region for the duration of one minute.

6. After one minute, we remove this cooling stress to allow the skin to re-warm at room temperature within 3-4 minutes (thermal recovery phase) (Fig. 1c-d).

7. During the thermal recovery phase, infrared images of the pigmented lesion are captured every 2 seconds (Fig. 1c-d).

8. All IR images (in addition to the white light and polarized light images) taken during the study are saved and stored using the Labview software.

\section{Image Processing}

1. The IR images are analyzed using a dedicated Matlab code in order to obtain accurate transient temperature distributions on the skin surface. For this purpose, we introduce several calibration steps and a multimodal image analysis system.

2. We start with applying a landmark detection algorithm to the bright light image for localizing the corners of the adhesive marker. Next, we identified the corresponding points in the reference IR image.

3. In order to compensate for involuntary body/limb movement of the patient, we use these points as landmarks in a quadratic motion model for aligning the IR image sequence during the recovery phase.

4. We use the random walker, an interactive image segmentation algorithm where the user can spatially guide the segmentation by placing seed points, to create a mask image delineating the lesion.

5. Once we determine the shape of the lesion, we identify the corresponding region in each of the registered IR images.

6. We select random points inside the lesion and away from the lesion representing the lesion and the healthy tissue, respectively.

7. We compare the transient thermal response of healthy skin and the response of the lesion.

8. We prepare a table showing all the data: digital, dermoscopy, color-coded IR images of the lesion and surrounding area recorded at ambient conditions and 2 seconds after the cooling excitation, and the transient thermal response of the lesion and the healthy tissue.

\section{Representative Results:}
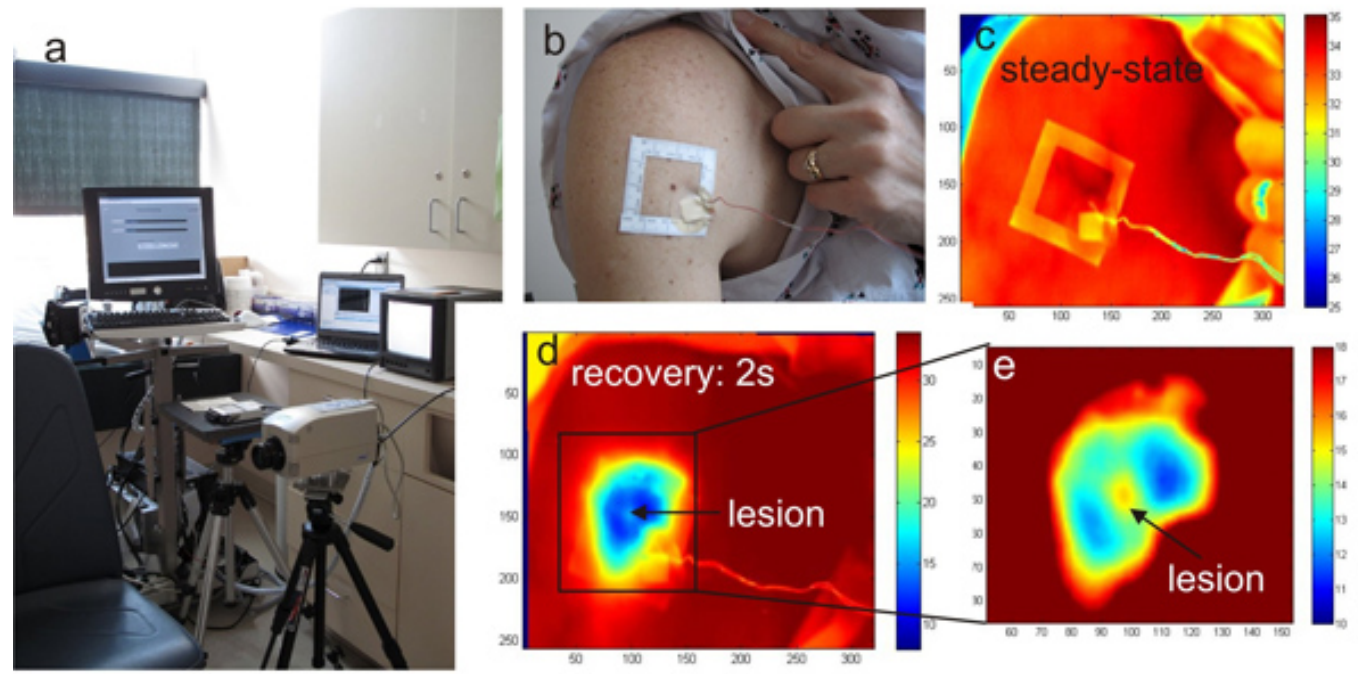

Figure 1. a) The infrared imaging system HRIS in the clinical trial room, b) photograph of the larger body surface area with a cluster of pigmented lesions and the template frame applied for imaging, c) reference infrared image of the region at ambient temperature, d) the same area after cooling and e) magnified section of the melanoma lesion and surroundings 


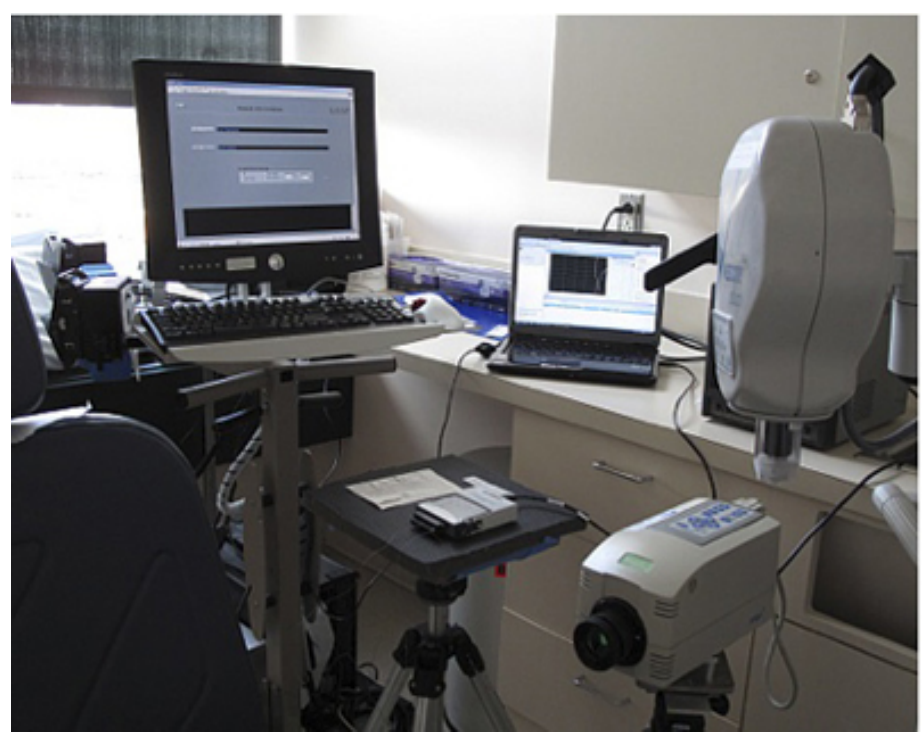

Figure 2. Exam room with our thermal imaging system.

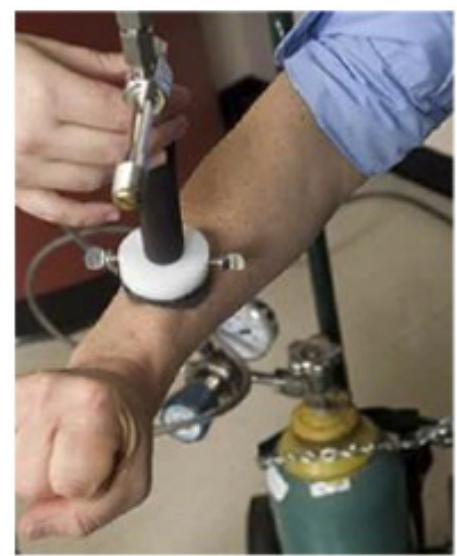

Figure 3. Cooling the lesion and the surrounding skin tissue by blowing a stream of cold air from a vortex tube.

\section{Discussion}

The results suggest that by applying the cooling stress we enhanced the temperature differences between the lesion and the surrounding healthy tissue. Also, because of small movements of the patient during the thermal imaging, we had to apply motion tracking to correctly overlay images to measure temperature differences between reference state and the temperature distribution during thermal recovery. Without the motion tracking we would not have been able to detect and measure the temperature difference between the malignant lesion and the healthy tissue. These results and the need for accurate motion tracking explain the difficulties investigators faced in the past when attempting to diagnose melanoma using IR imaging based on steady state information alone and clearly prove the advantages of dynamic thermal imaging.

It should be noted that the spatial resolution of the IR camera (number of pixels in the IR focal plane array) is critical when discerning small lesions. Both the spatial resolution and the temperature sensitivity of the early infrared cameras was limited, which also accounts for the difficulties in detecting early stage melanoma in the past. The key differences between our approach and prior thermal imaging attempts - that were moderately successful - are the sequences of calibration and image processing steps that allow us to accurately measure temperature differences in this system in addition to the dynamic imaging process that relies on active cooling.

\section{Disclosures}

No conflicts of interest declared.

\section{Acknowledgements}

This research was funded by the National Science Foundation Grant No. 0651981 and the Alexander and Margaret Stewart Trust though the Cancer Center of the Johns Hopkins University. The authors would like to acknowledge the contributions of Dr. Rhoda Alani to the IRB and the patient study as well as the help and support of Dr. Sewon Kang and his department during the patient study. 


\section{References}

1. Skin cancer foundation website (2010) [Online]. Available: http://www.skincancer.org/Skin-Cancer-Facts/.

2. Elder, D. Tumor progression, early diagnosis and prognosis of melanoma,.Acta. Oncol. 38, 535-547, (1999).

3. Wartman, D. \& Weinstock, M. Are we overemphasizing sun avoidance in protection from melanoma?. Cancer Epidemiol Biomarkers Prev. 17, 469-470, (2008).

4. Pirtini Cetingul, M. Using high resolution infrared imaging to detect melanoma and dysplastic nevi. Ph.D. dissertation, Johns Hopkins University (2010).

5. Jones, B. F. A reappraisal of the use of infrared thermal image analysis in medicine. IEEE Trans. Med. Imaging 17 (6), 1019-1027, (1998).

6. Anbar, M. Clinical thermal imaging today-shifting from phenomenological thermography to pathophysiologically based thermal imaging. IEEE Eng. Med. Biol. Mag. 17(4), 25-33, (1998).

7. Anbar, M., Gratt, B. M. \& Hong, D. Thermology and facial telethermography. Part I: history and technical review. Dentomaxillofacial Radiology 27, 61-67, (1998).

8. Jones, B. F. \& Plassmann, P. Digital infrared thermal imaging of human skin. IEEE Eng. Med. Bio. 21, 41-48, (2002).

9. Qi, H. \& Diakides, N. A. Infrared imaging in Medicine. CRC Press, (2007).

10. Pirtini Cetingul, M. \& Herman, C. Identification of skin lesions from the transient thermal response using infrared imaging technique. IEEE 5th Int. Symp. on Biomedical Imaging: From Nano to Macro 1-4, 1219-1222, (2008).

11. Pirtini Cetingul, M. and Herman, C., Quantification of the thermal signature of a melanoma lesion, Int. Journal of Thermal Science 50 , 421-431, (2011).

12. Pirtini Cetingul, M. \& Herman, C. A heat transfer model of skin tissue for the detection of lesions: sensitivity analysis. Physics in Medicine and Biology 55, 5933-5951, (2010)

13. Pirtini Cetingul, M. \& Herman, C. Quantitative evaluation of skin lesions using transient thermal imaging, Proc. Int. Heat Transfer Conf. IHTC14- 22465, (2010). 